\title{
Overexpression of folate binding protein and mesothelin are associated with uterine serous carcinoma ${ }^{\text {is }}$
}

\author{
Louis A. Dainty, John I. Risinger, Carl Morrison, G.V.R. Chandramouli, Michael A. Bidus, \\ Chris Zahn, G. Scott Rose, Jeff Fowler, Andrew Berchuck, G. Larry Maxwell* \\ Division of Gynecologic Oncology, Department of Obstetrics and Gynecology and the United States Military Cancer Institute, \\ Walter Reed Army Medical Center, Washington, DC 20307, USA \\ Laboratory of Biosystems and Cancer, Center for Cancer Research, National Cancer Institute, Bethesda, MD 20892, USA \\ Department of Laboratory Oncology Research, Curtis and Elizabeth Anderson Cancer Institute, Memorial Health University Medical Center, \\ Savannah GA 31404, USA \\ Department of Pathology and Division of Gynecologic Oncology, James Cancer Center, Ohio State University, Columbus, OH 43210, USA \\ Duke University Medical Center, Durham, NC 27110, USA
}

Received 23 January 2006

Available online 2 April 2007

\begin{abstract}
Purpose. Folate receptor alpha (FOLR1) is a membrane bound receptor involved in the transport of folate as well as other regulatory cellular processes. The purpose of this study was to examine the expression of FOLR1 in uterine cancers and to identify changes in gene expression that are associated with overexpression of FOLR1.

Experimental design. Fifty-eight frozen uterine cancer specimens were stained for FOLR1 using immunohistochemistry and results were correlated with transcript expression noted on quantitative PCR. Total RNA from 16 cases of uterine serous carcinoma (USC) was analyzed for gene expression using the Affymetrix HG-U133A and HG-U133B GeneChip set. USCs overexpressing FOLR1 were compared to cancers with an absence of FOLR1 using binary comparison and template matching of data was used to identify genes that correlate with FOLR1 expression. Selected targets from this analysis were evaluated by quantitative PCR as well as in an independent set of USC represented in quadruplicate on a tissue microarray (TMA).

Results. Overexpression of FOLR1 was observed in 11/16 (69\%) of USC and 0/10 normal endometrium cases using frozen tissue specimens. Binary comparison between FOLR1 positive and negative cases identified 121 genes altered by 2-fold at $p<0.01$ of which 45 are well correlated with FOLR1 expression pattern. Using quantitative PCR, both mesothelin (MSLN) and PTGS1 (COX1) were significantly increased in FOLR1 overexpressing tumors ( $p=0.014$ and $p=0.006$ respectively). TMA confirmed that overexpression of FOLR1 and MSLN respectively occurred in $23 / 48(48 \%)$ and $17 / 54(32 \%)$ of pure USC.

Conclusion. Both FOLR1 and MSLN are cell surface targets that are co-expressed at high levels in USC and are appealing targets for biologic therapy.
\end{abstract}

(C) 2007 Published by Elsevier Inc.

Keywords: Folate binding protein; Papillary serous; Gynecologic tumors

\footnotetext{
Presented at the 36th Annual Meeting of the Society of Gynecologic Oncologists, Miami, 2005.

* Corresponding author. Division of Gynecologic Oncology, Department of Obstetrics and Gynecology, Walter Reed Army Medical Center, 6900 Georgia Ave., NW, Washington, DC 20307, USA. Fax: +1 2027829278.

E-mail addresses: george.maxwell@na.amedd.army.mil, george.maxwell@us.army.mil (G.L. Maxwell).
}

\section{Introduction}

The folate receptor is a glycosol-phosphatidylinositol linked membrane protein that facilitates transfer of folate into the cell. Because folate provides the one-carbon units necessary for methylation of DNA, proteins, and phospholipids, cellular supply of folate can be important in the regulation of cellular processes [1]. Although a principal physiologic function of the folate receptor is to transport folate across the cellular membrane, 
it also may play a role in cellular proliferation, independent of its role in folate uptake [2,3]. The association between FOLR1 overexpression and unregulated cellular proliferation has not been elucidated in any significant detail. Multiple studies have demonstrated increased FOLR 1 expression in as many as $90 \%$ of ovarian cancers, particularly those of papillary serous histology [4-7]. However, there is limited information regarding the expression of FOLR1 in uterine cancer.

In a previous microarray study, we reported that the FOLR1 transcript was overexpressed in a significant proportion of endometrial adenocarcinomas [8]. Quantitative PCR indicated that the levels of FOLR1 transcript among uterine serous cancers (USC) were 60-fold greater than in normal endometrium. The purpose of the current investigation was to confirm whether overexpression of FOLR1, at both the transcript and protein level, occurs in USC and to determine which genes are typically co-expressed with FOLR1.

\section{Materials and methods}

\section{Frozen tissue specimens}

Fresh frozen tissue samples were obtained from 58 patients (16 uterine serous carcinomas, 13 endometrioid adenocarcinomas and 19 mixed mullerian tumors (MMMT) undergoing surgery for uterine cancer at Duke University Medical Center. Tissue specimens were evaluated by H\&E to confirm that the specimen to be analyzed contained greater than $50 \%$ cancer cells. During preparation of the specimens for analysis, care was taken to macroscopically dissect the cancers away from any adjacent myometrium. In addition, specimens of normal endometrium were obtained from age-matched women undergoing hysterectomy for benign indications. Normal endometrium was examined and glandular epithelium and stoma was grossly dissected from 10 of the specimens. All tissues were obtained after receiving consent described in a tissue banking process approved by that Institutions' Review Board (IRB) who granted authorization to collect and study the tissue samples.

\section{Immunohistochemistry for FOLR1 using frozen specimens}

Tissue specimens were cut into $5 \mu \mathrm{m}$ sections and mounted on untreated glass slides prior to fixation. After being re-hydrated and washed in buffers, the slides were incubated with the primary antibody (Mov 18/ZEL, Alexis Biochemicals) (diluted $1: 1000$ ) at room temperature for $3 \mathrm{~h}$. Antigen retrieval was not performed. Following washing in buffers, the slides were incubated with $0.3 \%$ Hydrogen peroxide in PBS for 40 min to block endogenous peroxidase activity. The slides were incubated with biotinylated horse antimurine immunoglobulin (Vector, Burlingame, CA, USA) and then with Vectastatin ABC Mix (Vector, Burlingame, CA, USA) at room temperature for $30 \mathrm{~min}$. Additional washing with buffers was performed prior to the slides being counterstained with Methyl Green. The slides then underwent a series of dehydration steps with $95 \%$ and $100 \%$ ethanol, and were then mounted with coverslips. Negative controls were used with each run and consisted of the ES-2 cell line, normal endometrium, and a PBS slide lacking MOV18 antibody. Positive controls included the SKOV-3 cell line, which had strong expression by Taqman analysis, and a set of 15 serous ovarian cancer specimens that were identified as being positive for FOLR1 during optimization of the immunohistochemical assays. The intensity of FOLR1 staining was graded from 0 to 3 and the proportion of tumor cells staining at each intensity level was also recorded. Cases in which at least $30 \%$ of the cells demonstrated a staining intensity of $2-3$ were considered positive.

\section{Microarray analysis}

The global gene expression for each of the 16 frozen USCs was assessed using the Affymetrix HG-U133A and HG-U133B (45,000 gene transcripts covering 28473 UniGene clusters) [9]. The global gene expression of these cases has been previously reported as part of a larger set of uterine cancers undergoing oligonucleotide microarray analysis [9]. This database was utilized to determine FOLR1 transcript expression for the 16 cases of uterine cancer as well as facilitate binary comparison and template matching according to FOLR1 expression.

Binary class comparison was used to compare global gene expression from samples with positive folate binding protein staining versus those without immunohistochemical staining. The analysis was performed using BRB Array tools software (BRB Array tools ver. 3.0c, Richard Simon, Amy Peng, Biometric research branch, NCI, NIH, http://inus.nci.nih.gov/BRB-ArrayTools. $\mathrm{html}$ ). Statistical calculations were performed on the logarithmic values of signals. Differentially expressed genes were identified by parametric Student's $t$-tests on genes having at least $5 \%$ or more present calls.

For Template Matching, 29272 genes having at least 1 present call in the serous samples were used for the analysis. Two templates were considered: one was idealized for FOLR1 positive and negative cases as +1 and -1 respectively; and the other was actual FOLR1 expression relative to normal endometria. The genes were selected if the correlation coefficient between either of the templates and gene expression pattern is $>0.7$ (or $\leq 0.7$ ), $t$-test $p<0.01$, and the ratio is 2 -fold for comparison between FOLR positive and negative cases. The expression data were displayed relative to average normal endometria expression. The cluster was color-coded using red for up-regulation from normal endometria and green for down-regulation.

\section{Validation of gene expression}

\section{Quantitative PCR}

The concentrations of select genes chosen for validation of gene expression were determined using the standard curve method for normalization and the results compared with average threshold PCR amplification cycle time $\left(C_{\mathrm{T}}\right)$ of normal endometrial samples. The relative gene expression (on logarithmic scale to base 2) of selected genes in each histologic group was compared to normal endometria. Gene expression assays for the analysis of samples were purchased from Applied Biosystems, Foster City, CA.

\section{Tissue microarray}

Paraffin tissue samples used for tissue microarray were collected from patients diagnosed with and treated for uterine malignancy between January 1st 1980 and July 31st 2003 at the Arthur James Cancer Hospital of the Ohio State University (OSU). The creation of the uterine cancer tissue microarray and use of this resource for the purposes of this study was performed following protocol approval by the OSU Institutional Review Board. An endometrial cancer TMA was constructed from the primary tumors harvested from the hysterectomy specimens of 485 patients. The specimens used for the tissue microarray represented various histologic types and stages of endometrial cancer. The presence of tumor tissue on the arrayed samples was verified on a hematoxylineosin-stained section and each case was represented in quadruplicate on the TMA. Specimens for controls consisted of 50 secretory endometrium, 50 proliferative endometrium, 50 normal cervix, and 50 normal ovaries.

Immunoperoxidase staining was performed on each formalin-fixed, paraffinembedded TMA cut at $4 \mu \mathrm{m}$ and placed on positively charged slides. Slides were placed in a $60^{\circ} \mathrm{C}$ oven for $1 \mathrm{~h}$, cooled, then deparaffinized and rehydrated using xylene and graded ethanol solutions to water. All slides were quenched for $5 \mathrm{~min}$ in a 3\% hydrogen peroxide solution in methanol to block for endogenous peroxidase. Antigen retrieval was performed by a steamer heat method in which the specimens were placed in a citric acid solution (Target Retrieval Solution, $\mathrm{pH}$ 6.1; Dako Cytomation, Carpinteria, CA), for $30 \mathrm{~min}$ at $94{ }^{\circ} \mathrm{C}$ using a vegetable steamer.

Multiple attempts were made to use the variations of the MOV-18 assay (used for initial staining of frozen tissue specimens) to evaluate FOLR1 expression in the paraffin tissue specimens on TMA without success. This antibody was robust in frozen tissue but is suboptimal in the staining of paraffin fixed tissue. Subsequent staining of the endometrial cancer TMA using the Pu-17 antibody (Endocyte Inc.) was successful in the staining of paraffin fixed tissues for FOLR1 expression. The Pu-17 antibody was diluted 1:50 and incubated for $60 \mathrm{~min}$. Immunohistochemical staining of MSLN was performed using (clone 5B2, Novocastra Laboratories) at a 1:10 dilution. All paraffin tissue slides underwent antigen retrieval in a vegetable steamer containing citrate buffer prior to being 
placed on a Dako Autostainer for immunohistochemical staining. Both FOLR1 and MSLN staining were graded using the following criteria: $0=$ no staining; $1=$ weak cytoplasmic staining, no membranous immunoreactivity; $2=$ moderate cytoplasmic staining with weaker membranous immunoreactivity; $3=$ strong cytoplasmic and membranous immunoreactivity. A case was considered as negative on TMA only if all four cores, representative of the case had no evidence of staining. The overall intensity of staining was recorded as that for the core with the strongest intensity when there was variation between cores for a given case. The results were recorded as positive if at least $10 \%$ of the neoplastic cells exhibited a staining grade of $2-3$. Correlation between FOLR1 and either MSLN or COX-1 expression was calculated using Cohen's kappa coefficient.

\section{Results}

Immunohistochemistry and quantitative PCR revealed that overexpression of both FOLR1 transcript and protein occurred in $11 / 16(69 \%)$ of uterine serous carcinomas 5/13 (38\%) endometrioid carcinomas and 7/19 (37\%) malignant mixed mullerian tumor. Staining was confined to the epithelial components of the uterine tissues that were examined. Correlations between transcript expression noted on quantitative PCR and protein expression observed using immunohistochemistry were noted for most of the individual cases. Expression of FOLR1 among normal endometrial specimens was minimal using quantitative PCR and was not observed in any of the specimens using immunohistochemistry (Fig. 1).

In order to assess if a group of genes distinguishes USC with increased FOLR1 expression from those with decreased FOLR1 expression, we performed a binary comparison of the expression data from these two groups. This analysis indicated that 245 array features were differentially expressed between cases with FOLR1 overexpression and those without (parametric Student's $t$-test $p$-value at $<0.005$ ) and that 121 of the 245 array features were differentially expressed by at least 2 -fold. The probability of finding at least 245 genes significant by chance if there are no real differences between the classes was 0.453 , implying that the that the global expression pattern between FOLR1 positive versus negative cases is not the principal delineating feature of these tumors. The complete data reflective of this analysis are also provided electronically (http://go.cancer.gov/12345).

Because FOLR1 is expressed in a significant proportion of uterine cancers, we used template matching in order to identify genes that could be co-expressed with FOLR1. Template matching revealed that 45 of 121 genes identified using binary comparison (differentially expressed by at least 2-fold at $p<0.005)$ correlated with FOLR1 expression in these tumors $(r>0.7$ or $r \leq 0.7$ at $p<0.01)$. This list of 46 transcripts (FOLR1+ the other 45 transcripts that are co-expressed with FOLR1) is shown in Fig. 2.

Using quantitative PCR, both mesothelin (MSLN) and PTGS1 (COX1) were significantly increased in FOLR1 overexpressing tumors ( $p=0.014$ and $p=0.006$ respectively). Overexpression of mesothelin correlated with FOLR1 overexpression $(r=0.74)$ while correlation of overexpression was marginal between COX1 and FOLR1 $(r=0.55)$ using the pilot set of frozen USC (Fig. 3).

A tissue microarray (TMA) was used to further validate FOLR1 and MSLN expression in uterine cancers (Figs. 4 and 5
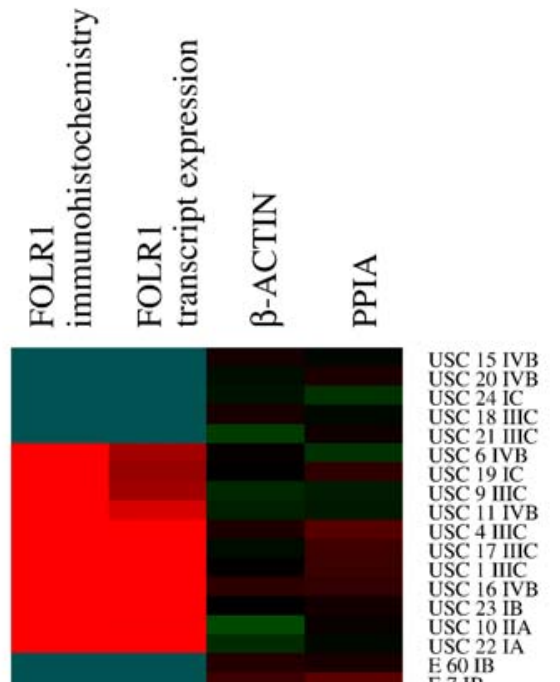

E 59 IIIC

E 61 IVB

E 62 IIIC

E 6 IB

E 53 IIIC

E 64 IB

E 36 IA

E 42 IA

MMMT 25 IIB

MMMT 17 IC

MMMT 10 IIIC

MMMT 26 IIIA

MMMT 13 IB
MMMT 14 IVB

MMMT 14 IVB
MMMT 27 IIIB

MMMT 2 IIIC

MMMT 12 IV

MMMT 7 IIIC

MMMT I IC

MMMT 3 IIB

MMMT 6 IA
MMMT 8 IIIC

MMMT 23 IC

MMMT 24 IIA

MMMT 28 IIIC

$\mathrm{N} 1$
$\mathrm{~N} 2$
$\mathrm{~N}$
$\mathrm{~N}$

N 10

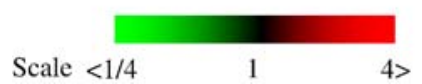

Fig. 1. Expression of FOLR1 in 58 frozen uterine cancer specimens (16 uterine serous carcinomas (USC), 13 endometrioid carcinomas (E), and 19 malignant mixed mullerian tumors [MMMT] $)$ and 10 specimens of normal endometrium using immunohistochemistry and quantitative PCR analysis. Expression of PPLA and $\beta$-actin were analyzed using quantitative PCR analysis in each of the cases and used as negative controls for assessment of FOLR1 transcript expression.

respectively). Differences in the total numbers of cases evaluated for FOLR1 and MSLN reflect differences in the transfer of histologic material from the TMA block to the slide. Of the 485 cases represented on the TMA, 475 cases were available for FOLR1 staining and 413 cases were available for MSLN staining. Immunohistochemical staining revealed overexpression of FOLR1 in 68/411 (16\%) uterine cancers represented on the tissue microarray and varied by histology: 23/48 (48\%) pure serous; 7/25 (28\%) mixed epithelial; 2/7 (28\%) clear cell; and 4/ 25 (16\%) MMMT. Overexpression in endometrioid carcinomas was noted in $33 / 308(18 \%)$ cases and appeared to be less often 


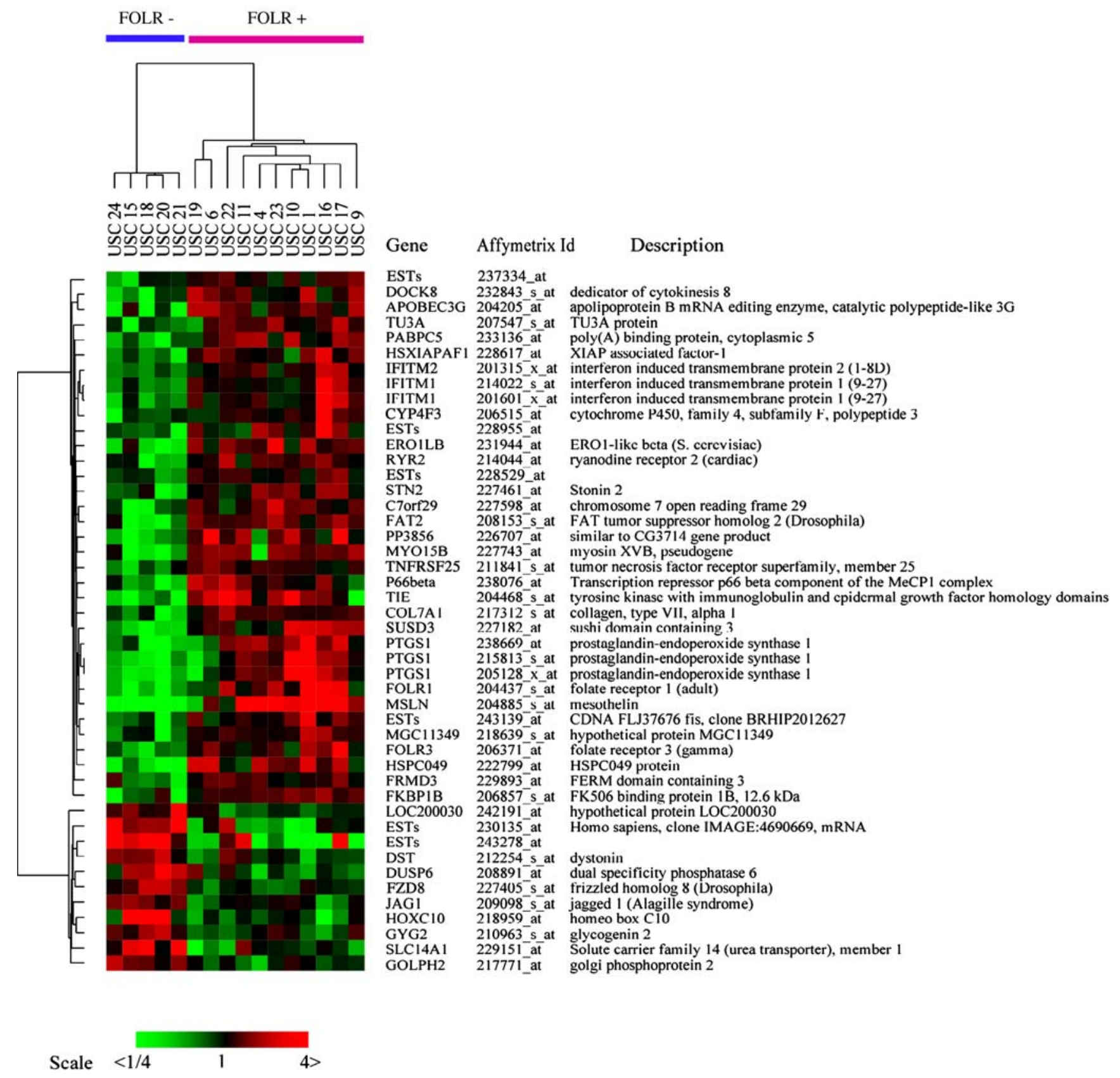

Fig. 2. A binary comparison of 16 frozen USC (5 FOLR - and 11 FOLR1+) revealed 121 genes that are differentially expressed by at least 2 -fold at $p<0.005$. Template matching analysis of these data provided a list of 45 other transcripts that are co-expressed with FOLR1. Expressions are mean centered with a distance metric: 1-correlation. The heat map was color-coded using red for up-regulation from normal endometria and green for down-regulation. The data clusters according to FOLR immunohistochemical staining status of the 16 frozen specimens (green for negative and red for positive) noted at the top of the heatmap.

observed in G3 tumors (G1, 15/144 [11\%]; G2, 15/107 [14\%]; and G3 3/57 [5\%]) (Table 1). Immunohistochemical staining of mesothelin revealed overexpression in approximately $20 \%$ of the uterine cancers: $17 / 54(32 \%)$ pure serous; $5 / 26(20 \%)$ mixed epithelial; $1 / 7$ (14\%) clear cell; 6/28 (22\%) MMMT; and endometrioid 66/360 (18\%). Mesothelin expression appeared to be independent of grade in the subset of endometrioid endometrial carcinomas) (Table 2). Correlation of FOLR1 expression with MSLN expression on the TMA revealed agreement $73 \%$ of the time $(p=0.02)$.
Expression of FOLR1 was also correlated with COX-1 staining, which was reported previously [10]. In $80 \%$ of cases, FOLR1 overexpression was accompanied by COX-1 overexpression $(p=0.0001)$.

\section{Discussion}

Overexpression of FOLR1 in uterine cancers has been demonstrated previously using a variety of antibodies has been reported previously, but the scope of prior studies, but 
PTGS1

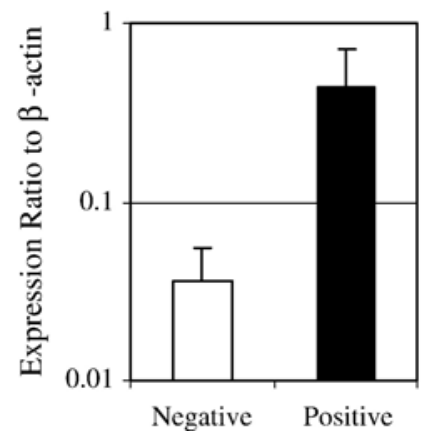

MSLN

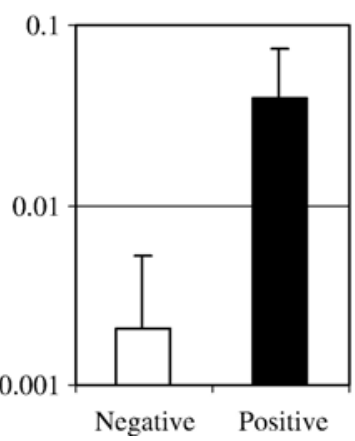

FOLR1

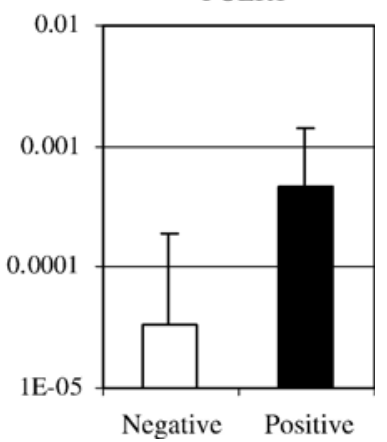

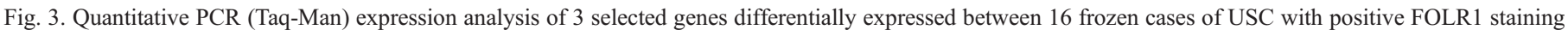

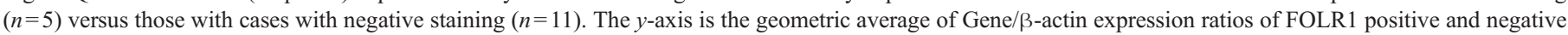
samples.

the scope of prior studies has been limited by the small number of cases examined. Compiling the results from 4 of these studies, overexpression of FOLR1 was observed in a total of 31/46 cases of endometrial adenocarcinoma [11-14]. The relationship between FOLR1 overexpression and USC was not reported in any of the previous studies, so it is unclear whether this is a notable feature of USC.

Similar to previous reports, we found that FOLR1 staining of endometrial cancers was confined to epithelial component of the tumor and staining was not seen in stromal elements. In addition, FOLR1 overexpression was not observed in the sarcomatous components of the malignant mixed mullerian tumors. In our current analysis, we observed that FOLR1 is overexpressed at the transcript and protein level in a significant proportion $(11 / 18$, $69 \%$ ) of USC compared to normal endometrium (0/10). Further validation of overexpression using TMA revealed that FOLR1 was overexpressed in $23 / 48$ (48\%) pure USC compared to $7 / 25$ $(28 \%)$ of mixed epithelial carcinomas with serous components.

The function of FOLR in USC remains to be elucidated. Previous reports involving papillary serous cancers of the ovary have noted an indirect relationship between expression of FOLR and Caveolin-1 (CAV-1) (a putative tumor suppressor gene)
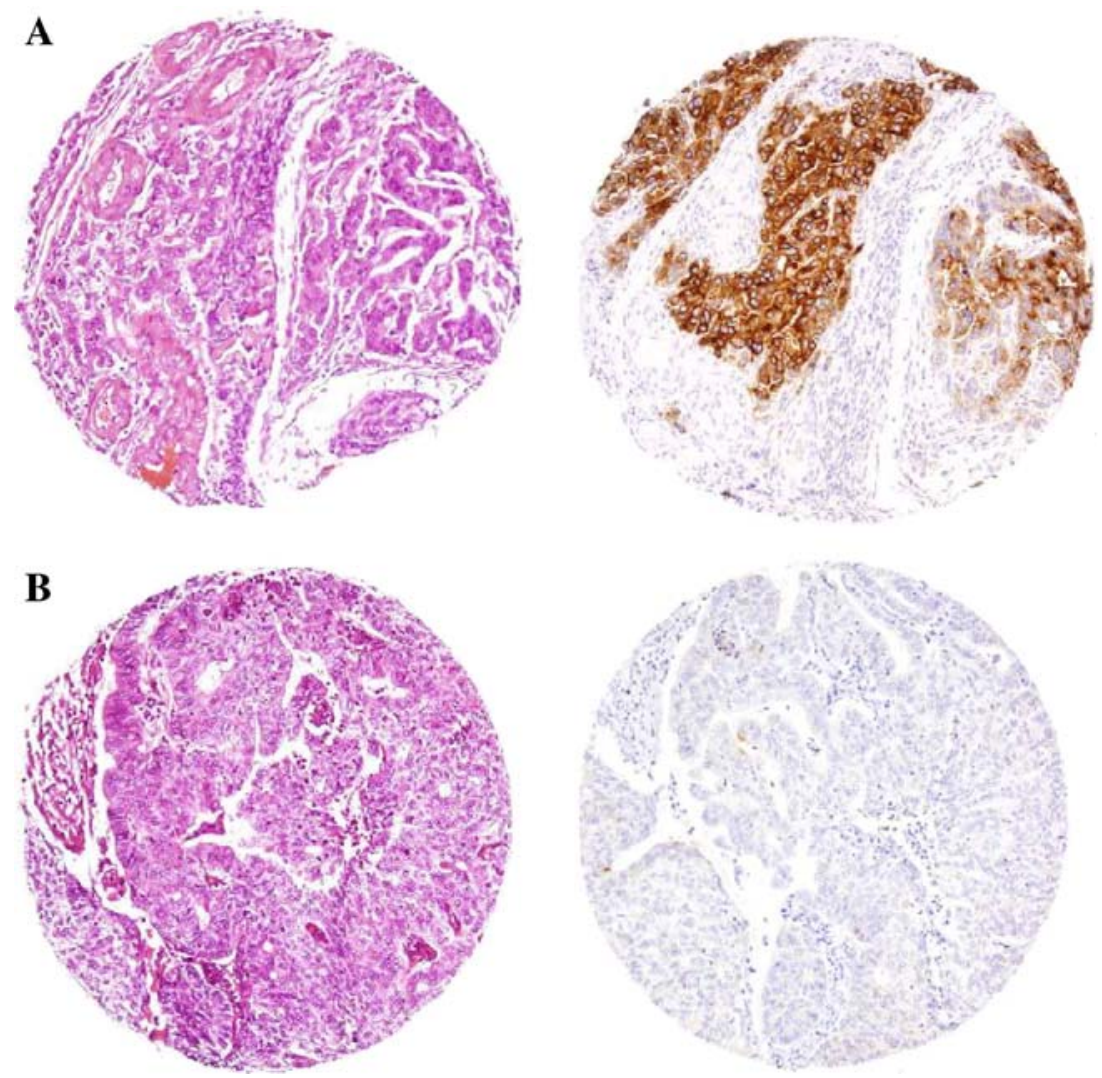

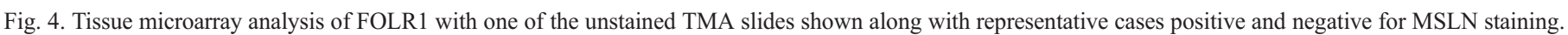
The top panel demonstrates an example of uterine serous carcinoma and the bottom panel represents an endometrioid carcinoma. 

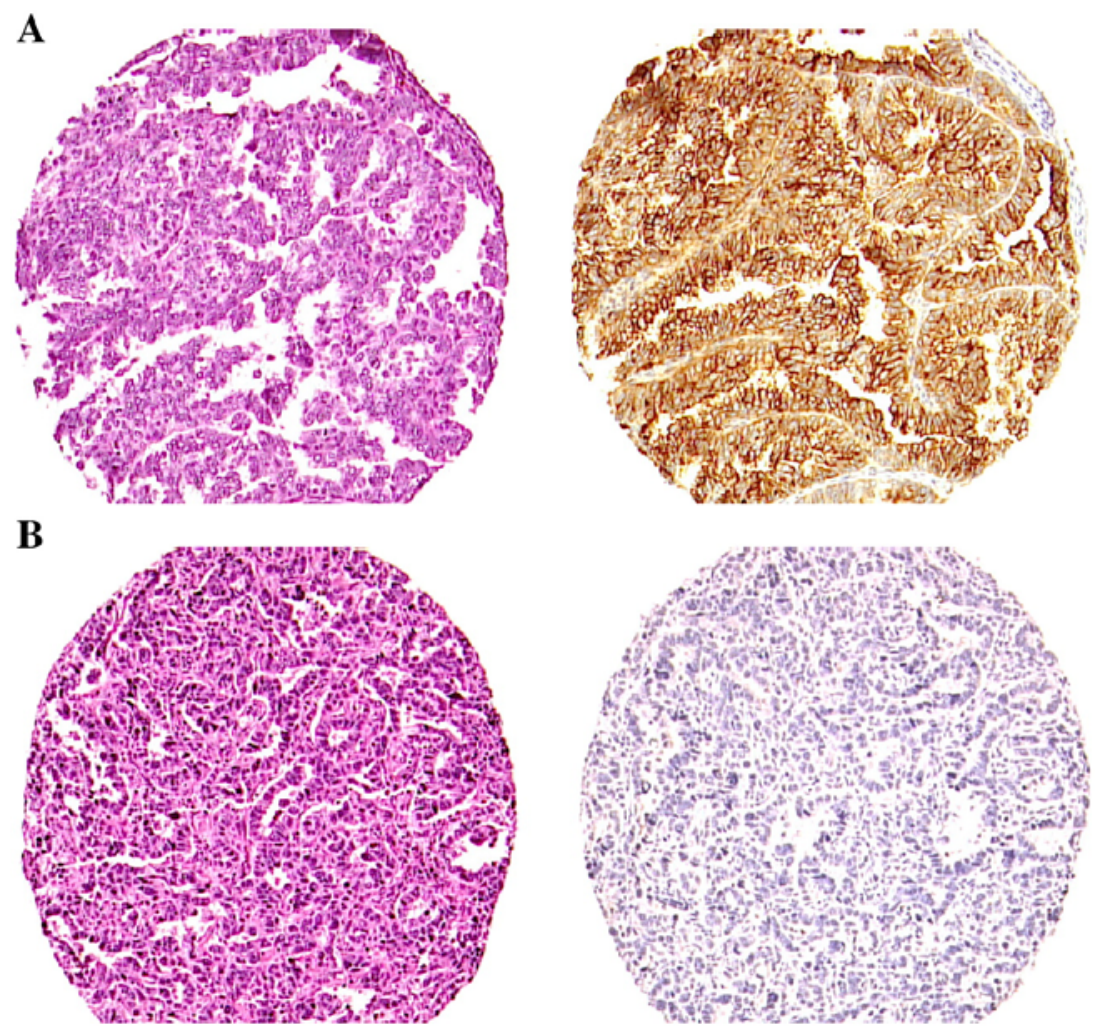

Fig. 5. Tissue microarray analysis of mesothelin with one of the unstained TMA slides shown along with representative cases positive and negative for MSLN staining. The top panel demonstrates an example of uterine serous carcinoma and the bottom panel represents an endometrioid carcinoma.

$[15,16]$. Likewise microarray data from our group involving an analysis of uterine carcinomas [9] has suggested that CAV-1 expression may be increased in papillary serous and high grade endometrioid endometrial carcinomas. However, correlation of CAV and FOLR1 expression was not evaluated in this prior study. In the current analysis, co-expression of CAV-1 with FOLR1 was not observed using template matching. This suggests that if CAV-1 expression is low in some USC it may not be an event that is inherently related to FOLR1 expression as in vivo studies of ovarian papillary serous carcinomas [15] have indicated.

The overexpression of FOLR1 in cancerous tissues compared to minimal expression in their normal tissue counterparts has prompted development of receptor-targeted molecular therapies. High affinity binding of folic acid conjugates to FOLR1 facilitates delivery of molecular therapies at low concentrations [17]. In addition, FOLR1 that is present in normal tissues is usually localized to the apical surfaces of the epithelium, which leads to a low toxicity profile associated with systemic therapy $[17,18]$. Although folate conjugates can localize to the apical surfaces of proximal renal tubules, they remain contained within lysosomes following endocytosis and are transported across the nephron to the basolateral side of the epithelium. In contrast, cytosolic release of folate conjugates primarily occurs in neoplastic cells [19]. These alternate methods of processing folate between cancer cells and normal cells may serve to minimize the potential for renal toxicity while maintaining effective cytotoxicity against neoplastic tumors. Recent data from a Phase I trial involving a folate acid conjugate has not revealed evidence of renal toxicity [20].

FOLR1 staining has been reported to be increased in the majority of ovarian cancers, particularly those of papillary serous histology [10]. Levels of FOLR1 in these neoplastic tissues can be increased as high as 80-90 fold compared to normal ovarian epithelium [6]. Immunogenic FOLR1 peptides that elicit Cytotoxic T Lymphocyte (CTL) response have been developed for the use in ovarian carcinoma [13,21-23] and could potentially be used in the adjuvant treatment of USC that overexpress this gene and protein. Anecdotally, we observed that the intensity of staining was less intense among USC compared to the papillary serous cancers of the ovary used as positive controls. Use of antiestrogens may enhance the effects of adjuvant therapies targeting FOLR1 because of the temporarily

Table 1

Immunohistochemical staining of an endometrial cancer tissue microarray for FOLR1 (using Pu-17)

\begin{tabular}{lccc}
\hline Histologic subtypes & Total no. & No. FOLR1+ & \% FOLR1+ \\
\hline Endometrioid G1 & 144 & 15 & 10.42 \\
Endometrioid G2 & 107 & 15 & 14.02 \\
Endometrioid G3 & 57 & 3 & 5.26 \\
Mixed epithelial & 25 & 7 & 28.00 \\
MMMT & 25 & 4 & 16.00 \\
Serous & 48 & 23 & 47.92 \\
Clear cell & 7 & 2 & 20.00 \\
Total cases & 413 & 69 & 16.70 \\
\hline
\end{tabular}


Table 2

Immunohistochemical staining of an endometrial cancer tissue microarray for MSLN

\begin{tabular}{lccl}
\hline Histologic subtypes & Total no. & No. MSLN+ & \% MSLN+ \\
\hline Endometrioid G1 & 182 & 36 & 19.78 \\
Endometrioid G2 & 114 & 21 & 18.42 \\
Endometrioid G3 & 64 & 9 & 14.06 \\
Mixed Epithelial & 26 & 5 & 19.23 \\
MMMT & 28 & 6 & 22.22 \\
Serous & 54 & 17 & 31.48 \\
Clear cell & 7 & 1 & 14.29 \\
Total cases & 475 & 95 & 19.96 \\
\hline
\end{tabular}

elevated levels of FOLR1 that can result from this complementary therapy [24].

Using binary comparison analysis, we found that the global gene expression pattern between FOLR1 positive versus negative cases is not the principal delineating feature of these USC. In an effort to identify genes co-expressed with FOLR1 that may be potential molecular markers associated with USC, we performed template matching using the gene expression data representative of this subset of uterine cancers. In this analysis, we found that overexpression of MSLN was more often associated with USC that displayed FOLR1 overexpression. In an analysis of 150 carcinomas from various anatomic sights, Frierson et al. [25] noted increased expression of mesothelin in $13 / 22(64 \%)$ of endometrioid endometrial adenocarcinomas. Although this study did not include USC, MSLN overexpression was noted in all 38 serous carcinomas of the ovary that were evaluated [25]. Our study showed overexpression of MSLN in 17/54 (32\%) pure USC and 66/360 (18\%) endometrioid endometrial carcinomas represented on the TMA.

Although overexpression of MSLN has not previously been observed in USC, increased MSLN has been noted in mesotheliomas, as well as carcinomas of the pancreas, stomach, lung, esophagus, cervix, endometrium, and ovary, [26,27]. Mesothelin is a glycosyl-phosphatidylinositol-linked cell surface antigen that is expressed by mesothelial cells lining the abdominal, pelvic, and pleural cavities. Recently investigators have hypothesized that metastasis of ovarian cancer cells may be facilitated through binding of CA-125 coated ovarian cancer cells to MSLN in the peritoneal epithelium [28]. The potential interaction between MSLN and FOLR1 in USC tumor cells is unknown.

Microarray experiments have suggested that there are global differences in gene expression between serous carcinomas of uterine versus ovarian origin [29]. However, there still may be similarities in expression of certain genes that account for the aggressive clinical behavior associated with serous tumors that arise in both organs. Overexpression of MSLN (which occurs in approximately $75 \%$ of ovarian serous carcinomas) was found to be increased in $32 \%$ of USC evaluated using TMA. It is unknown whether MSLN could contribute to an "ovarian like" pattern of peritoneal metastasis cancer that characterizes some USC. Our TMA contained too few cases of stage IV USC to definitively investigate this hypothesis. However, there were no obvious differences in the degree of overexpression between those USC patients with stage IV disease $(3 / 13,23 \%)$ and those patients with all other stages of disease $(16 / 45,35 \%)$.

The significance of mesothelin overexpression in specific human cancers has led to the development of mesothelin as a potential therapeutic target. Hassan et al. [30] have used a form of Pseudomonas exotoxin bound to monoclonal immunoglobulin that recognizes mesothelin to induce complete regression of mesothelin positive human cancer xenographs in mice. More recently, a recombinant antimesothelin immunotoxin has been developed and shown to be cytotoxic to mesothelin expressing human ovarian and cervical tumors of varying histologic subtypes [31]. Mesothelin is currently being investigated as a mediator in adenovirus vectors for ovarian cancer gene therapy [32].

Our data also showed that COX-1 expression was increased in USC characterized by FOLR1 overexpression. Both COX-1 and COX-2 are associated with production of prostaglandins [33], which have been implicated in both and angiogenesis and carcinogenesis [34]. However, the association of COX-1 overexpression with increased FOLR1 has not explored in detail. Dore et al. [35] demonstrated that COX-1 is expressed in ovarian surface epithelium as well as ovarian adenocarcinomas including papillary serous cases. Likewise, COX-1 was investigated in 60 non-endometrioid endometrial cancers represented on a tissue microarray composed of 336 endometrial cancers and specimens of normal endometrium from 105 patients. In this analysis, COX-1 expression was observed in approximately $25 \%$ of both normal endometrium and nonendometrioid endometrial cancer while COX-2 expression was found on approximately $60 \%$ of non-endometrioid tumors (a 2 fold increase in frequency compared to normal endometrium cases) [10]. These data would suggest that although COX-1 expression is associated with tumors having increased FOLR1, the expression of COX-1 in normal endometrium precludes it from being an ideal target for chemoprevention of USC. COX-1 is constitutively expressed in many tissues and may be necessary for normal physiologic processes [36].

Although less than $10 \%$ of endometrial cancers are USC, almost $50 \%$ of endometrial cancer recurrences and deaths are attributable to these virulent tumors [37]. In view of the poor efficacy of current treatment modalities, identification of novel therapeutic approaches is a high priority. Vaccine and molecular therapies targeting either FOLR1 or MSLN are currently being tested in Phase I trials in other types of solid tumors [38,39]. The findings of the present study that FOLR1 and MSLN are overexpressed in $48 \%$ and $43 \%$ of USC suggests that these cancers should be included in future clinical trials targeting FOLR1 and MSLN.

\section{Acknowledgments}

This study was funded by the Department of Defense Peer Reviewed Medical Research Program, grant \#PR012331. The opinions expressed in this article represent the private views of the authors and should not be construed as reflecting the official views of the Department of Defense. The opinions or assertions contained herein are the private views of the authors and are not 
to be construed as official or as reflecting the views of the Department of the Army or the Department of Defense.

\section{References}

[1] Jacob JA. Folate, DNA methylation, and gene expression: factors of nature and nurture. Am J Clin Nutr 2000;72:903-4.

[2] Corona G, Giannini F, Fabris M, Toffoli G, Boiocchi M. Role of folate receptor and reduced folate carrier in the transport of 5-methyltertahydrofolic acid in human ovarian carcinoma cells. Int J Cancer 1998;75: $125-33$.

[3] Toffoli G, Cernigoi C, Russo A, Gallo A, Bagnoli M, Boicchi M. Overexpression of folate binding protein in ovarian cancers. Int J Cancer 1997; 74:193-8.

[4] Campbell IG, Jones TA, Foulkes WD, Trowsdale J. Folate-binding protein is a marker for ovarian cancer. Cancer Res 1991;51:5329-38.

[5] Mantovani LT, et al. Folate binding protein distribution in normal tissues and biological fluids from ovarian carcinoma patients as detected by the monoclonal antibodies MOv18 and MOv19. Eur J Cancer 1994;30A: 363-9.

[6] Li PY, Vecchio SD, Fonti R, Carriero MV, Potena MI, Botti G, et al. Local concentration of folate binding protein GP38 in sections of human ovarian carcinoma by in vitro quantitative autoradiography. J Nucl Med 1996;37: $665-72$.

[7] Buist MR, Molthoff CFM, Kenemans P, Meijer GJLM. Distribution of OV-TL 3 and MOv18 in normal and malignant ovarian tissue. J Clin Pathol 1985;48:631-6.

[8] Risinger JI, Maxwell GL, Chandramouli GV, Jazaeri A, Aprelikova O, Patterson T, et al. Microarray analysis reveals distinct gene expression profiles among different histologic types of endometrial cancer. Cancer Res 2003;63:6-11.

[9] Maxwell GL, Chandramouli G, Dainty L, Litzi T, Berchuck A, Barrett JC, et al. Microarray analysis of endometrial carcinomas and mixed mullerian tumors reveals distinct gene expression profiles associated with different histologic types of uterine cancer. Clin Cancer Res 2005;11:4056.

[10] Fowler JM, Ramirez N, Cohn DE, Kelbick N, Pavelka J, Ben-Shacher I, et al. Correlation of cyclooxygenase-2 (COX-2) and aromatase expression in human endometrial cancer: tissue microarray analysis. Am J Obstet Gynecol 2005;192:1262-7.

[11] Wu M, Gunning W, Ratnam M. Expression of folate receptor type $\alpha$ in relation to cell type, malignancy, and differentiation in ovary, uterus, and cervix. Cancer Epidemiol Biomark Prev 1999;8:775-82.

[12] Garin-Chesa P, Campbell I, Saigo PE, Lewis JL, Old LJ, Rettig WJ. Trophoblast and ovarian cancer antigen LK26.: sensitivity and specificity in immunopathology and molecular identification as a folate binding protein. Am J Pathol 1993;142:557-67.

[13] Boerman OC, van Niekerk CC, Makkink K, Hanselaar TGJM, Kenemans P, Poels LG. Comparative immunohistochemical study of four monoclonal antibodies directed against ovarian carcinoma-associated antigens. Int J Gynecol Pathol 1991;10:15-25.

[14] Kim DK, Lee TV, Castilleja A, Anderson BW, Peoples GE, Kudelka AP, et al. Folate binding protein peptide 191-199 presented on dendritic cells can stimulate CTL from ovarian and breast cancer patients. Anticancer Res 1999;19:2907-16.

[15] Bagnoli M, Canevari S, Figini M, Mezzanzanica D, Raspagliesi F, Tomassetti A, et al. A step further in understanding the biology of the folate receptor in ovarian carcinoma. Gynecol Oncol 2003;88:S140-4.

[16] Bagnoli M, Tomassetti A, Figini M, Flati S, Dolo V, Canevari S, et al. Downmodulation of caveolin-1 expression in human ovarian carcinoma is directly related to $\alpha$-folate receptor overexpression. Oncogene 2000;19: 4754-63.

[17] Low PS. Folate receptor-targeted drugs for cancer and inflammatory diseases. Adv Drug Deliv Rev 2004;56:1055-8.

[18] Ross JF, Chaudhuri PK, Ratnam M. Differential regulation of folate receptor isoforms in normal and malignant tissues in vivo and in established cell lines. Physiologic and clinical implications. Cancer 1994;73: 2432-43.
[19] Sandoval RM, Kennedy MD, Low PS, Molitoris BA. Uptake and trafficking of fluorescent conjugates of folic acid in intact kidney determined using intravital two-photon microscopy. Am J Cell Physiol 2004;287:C257-9.

[20] Amato RJ, Engel JL, Low P, Naveed F. Phase I trial of EC90 (keyholelimpet hemocyanin fluorescein isothiocyanate conjugate) with GPI-0100 adjuvant followed by EC 17 (folate-fluorescein isothiocyanate conjugate) in patients with metastatic renal cell carcinoma and ovarian cancer. Abstract in the 2004 ASCO Annual Meeting Proceedings, vol. 22. JCO; 2004. p. 4754.

[21] Peoples GE, Anderson BW, Lee TV, Murray JL, Kudelka AP, Wharton JT, et al. Vaccine implications of folate binding protein, a novel cytotoxic T lymphocyte-recognized antigen system in epithelial cancers. Clin Cancer Res 1999;5:4214-23.

[22] Peoples GE, Anderson BW, Fisk B, Kudelka AP, Wharton JT, Ioannides CG. Ovarian cancer-associated lymphocyte recognition of folate binding protein peptides. Ann Surg Oncol 1998;5:743-50.

[23] Canevari S, Stoter G, Arienti F, Bolis G, Colnaghi MI, Di Re EM, et al. Regression of advanced ovarian carcinoma by intraperitoneal treatment with autologous T-lymphocytes retargeted by a specific monoclonal antibody. J Natl Cancer Inst 1995;87:1463-9.

[24] Kelley KMM, Rowan BG, Ratnam M. Modulations of the folate receptor $\alpha$ gene by the estrogen receptor: mechanism and implications in tumor targeting. Cancer Res 2003;63:2820-8.

[25] Frierson HF, Moskaluk CA, Powell SM, Zhang H, Cerilli LA, Stoler MH, et al. Large-scale molecular and tissue microarray analysis of mesothelin expression in common human carcinomas. Hum Pathol 2003;34:605-9.

[26] Hough CD, Sherman-Baust CA, Pizer ES, Montz FJ, Im DD, Rosensheir $\mathrm{NB}$, et al. Large-scale serial analysis of gene expression reveals genes differentially expressed in ovarian cancer. Cancer Res 2000;60:6281-7.

[27] Cao D, Ji H, Ronnett BM. Expression of mesothelin, fascin, and prostate stem cell antigen in primary ovarian mucinous tumors and their utility in differentiating primary ovarian mucinous tumors from metastatic pancreatic mucinous carcinomas in the ovary. Int J Gynecol Pathol 2005;24:67-72.

[28] Rump A, Morikawa Y, Ranaka M, Minami S, Umesaki N, Takeuchi M, et al. Binding of ovarian cancer antigen CA125/MUC16 to mesothelin mediates cell adhesion. J Biol Chem 2004;279(10):9190-8.

[29] Zorn KK, Bonome T, Gangi L, Chandramouli GV, Awtrey GS, Gardner GJ, et al. Gene expression profiles of serous, endometrioid, and clear cell subtypes of ovarian and endometrial cancer. Clin Cancer Res 2005;11: 6422-30.

[30] Hassan R, Viner JL, Wang QC, Marguiles I, Kreitman RJ, Pastan I. Antitumor activity of K1-LysPE38QQR, an immunotoxin targeting mesothelin, a cell-surface antigen overexpressed in ovarian cancer and malignant mesothelioma. J Immunother 2000;23:473-9.

[31] Hassan R, Lerner MR, Benbrook D, Lightfoot SA, Brackett DJ, Wang QQ, et al. Antitumor activity of SS(dsFv)PE38 and SS1(dsFv)PE38, recombinant antimesothelin immunotoxins against human gynecologic cancers grown in organotypic culture in vitro. Clin Cancer Res 2002;8:3520-6.

[32] Breidenbach M, Rein DT, Everts M, Glasgow JN, Wang M, Passineau M, et al. Mesothelin-mediated targeting of adenoviral vectors for ovarian cancer gene therapy. Gene Ther 2005;12:187-93.

[33] Smith WL, Langenbach R. Why are there two cyclooxygenase isozymes? J Clin Invest 2001;107:1491-5.

[34] Gupta RA, Tejada LV, Tong BJ, Das SK, Morrow JD, Dey SK, et al. Cyclooxygenase-1 is overexpressed and promotes angiogenic growth factor production in ovarian cancer. Cancer Res 2003;63:906-11.

[35] Dore M, Cote LC, Mitchell A, Sirois J. Expression of prostaglandin G/H synthase type 1, but not type 2, in human ovarian adenocarcinomas. J Histochem Cytochem 1998;46:77-84.

[36] Smith WL, Langenbach R. Why are there two cyclooxygenase isozymes? J Clin Invest 2001;107:1491-5.

[37] Hendrickson M, Ross J, Eifel P, Cox RS, Martinez A, Kempson R. Uterine serous carcinoma: a highly malignant form of endometrial adenocarcinoma. Am J Surg Pathol 1982;6:93-108.

[38] http://www.clinicaltrials.gov/ct/ScreenAdvancedSearch.

[39] www.sabin.org/PDF/LUL_0705.pdf. 